\title{
ENVIRONMENTAL AND GENOTYPE-ENVIRONMENTAL COMPONENTS OF VARIABILITY \\ IV. NON-LINEAR INTERACTIONS FOR MULTIPLE INBRED LINES
}

\author{
JEAN M. PERKINS and J. L. JINKS \\ Department of Genetics, University of Birmingham
}

Received 10.i.67

\section{Introduction}

WHILE a significant proportion of the genotype-environmental interaction component of variation is a linear function of the additive environmental component, there is frequently a significant remainder that is not (Perkins and Jinks, 1968). In the present paper the nature of this non-linear component of variation will be investigated. Two approaches will be used. In the first the genotypes will be separated into groups consisting of lines which are relatively homogeneous in their reactions to environmental differences to see whether or not this leads to a reduction in the non-linear component of the interaction. In addition, we shall examine the relationships between these groupings and other measurable differences among the genotypes. In the second approach the basis of the variation for the magnitude of the nonlinear component of the genotype-environmental interaction among the genotypes will be examined.

\section{THEORY}

In the model outlined in a previous paper of this series (Perkins and Jinks, 1968) in which the genotype-environmental interaction component for each line in each environment is expressed as a linear function of the additive environmental component, the deviation from the regression line for each line in each environment is treated as a fixed rather than a random effect. Hence the remainder M.S. after fitting the linear regression is tested for significance against an error M.S. derived from the variation within each line in each environment averaged over all lines and environments.

Correlations can be obtained for the deviations from the linear regressions over environments for each pair of lines to assess the relative similarities in their interactions with environmental differences, which are not accounted for by the linear component. There will be no correlation if the direction and magnitude of the deviation in each environment is independent for each of two lines. The correlation will be significant and positive if the deviations in each environment are predominantly in the same direction and have the same relative magnitudes for the two lines, or significant and negative if the deviations are predominantly in opposite directions to the same relative degree.

Thus following Perkins and Jinks (1968), we can write the expected performance of the $i$ th line in the $j$ th environment as

$$
r_{i j}=\mu^{\prime}+d_{i}^{\prime}+\epsilon_{j}^{\prime}+\beta_{i}^{\prime} \epsilon_{j}^{\prime}+\delta_{i j}
$$


where

$$
g_{i j}^{\prime}=\beta_{i}^{\prime} \epsilon_{j}^{\prime}+\delta_{i j}
$$

that is, the genotype-environmental interaction component, $g_{i j}^{\prime}$, consists of the linear portion, $\beta_{i}^{\prime} \epsilon_{j}^{\prime}$ and the deviation from regression, $\delta_{i j}$. The items in the analysis from the regression of $g_{i j}^{\prime}$ on $\epsilon_{j}^{\prime}$ for the $i$ th line have the following expectations :

$$
\begin{aligned}
& \text { Regression M.S. }=\frac{\left(\sum_{j} g_{i j}^{\prime} \epsilon_{j}^{\prime}\right)^{2}}{\sum_{j}\left(\epsilon_{j}^{\prime}\right)^{2}} \\
& =\left(\beta_{i}^{\prime}\right)^{2} \sum_{j}\left(\epsilon_{j}^{\prime}\right)^{2} \quad d f=1 \\
& \text { Remainder M.S. }=\frac{\sum_{j}^{\sum} \delta_{i j}^{2}}{s-2} \quad d f=s-2
\end{aligned}
$$

where $s=$ number of environments.

The items for an analysis of covariance between the $g_{i j}^{\prime}$ values for any pair of lines taken from a range of inbred lines, for example $i=1$ and 2 respectively, are as follows:

$$
\begin{aligned}
\text { Regression Cov. } & =\frac{\sum_{j}^{\sum}\left(g_{1 j}^{\prime} \epsilon_{j}^{\prime}\right)\left(g_{2 j}^{\prime} \epsilon_{j}^{\prime}\right)}{\sum_{j}\left(\epsilon_{j}^{\prime}\right)^{2}} & \\
& =\beta_{1}^{\prime} \beta_{2}^{\prime}{\underset{j}{\Sigma}\left(\epsilon_{j}^{\prime}\right)^{2}}^{\sum} & \\
\text { Remainder Cov. } & =\frac{\sum_{j} \delta_{1 j} \delta_{2 j}}{s-2} & d f=1
\end{aligned}
$$

As in the normal linear regression analysis the remainder item, that is, the sum of cross-products of $\delta_{1}$; and $\delta_{2 j}$, can be derived as the difference between the total S.C.P. and the regression S.C.P. where

$$
\begin{aligned}
& \text { Total S.C.P. }=\sum_{j} g_{1 j}^{\prime} g_{j}^{\prime} \\
&=\sum_{j}\left(\beta_{1}^{\prime} \epsilon_{j}^{\prime}+\delta_{1 j}\right)\left(\beta_{2}^{\prime} \epsilon_{j}^{\prime}+\delta_{2 j}\right) \\
&=\beta_{1}^{\prime} \beta_{2}^{\prime} \sum_{j}^{\Sigma}\left(\epsilon_{j}^{\prime}\right)^{2}+\sum_{j} \delta_{1 j} \delta_{2 j} \\
& \text { since } \beta_{1}^{\prime} \sum_{j} \epsilon_{j}^{\prime} \delta_{2 j}=\beta_{2}^{\prime} \underset{j}{\Sigma} \epsilon_{j}^{\prime} \delta_{1 j}=0
\end{aligned}
$$

The correlation, $r$, for the deviations from the linear regression for lines 1 and 2 therefore has the following form:

$$
r=\frac{\sum_{j}^{\sum} \delta_{1} \delta_{2 j}}{\sqrt{\sum_{j} \delta_{1 j}^{2} \sum_{j} \delta_{2 j}^{2}}}
$$

A correlation matrix can be computed in which the correlation between pairs of lines in every combination is represented. Each will in fact be represented twice since the correlation between lines 1 and 2 is the same as 
that between 2 and 1. Some of the correlations may be significant and positive while others may be significant and negative. As will be shown in the next section, the lines can be separated into groups, which are internally homogeneous, on the basis of the pattern of the significant correlations between them. According to the character and set of lines under study, a few such distinct groups may or may not emerge.

Additive environmental values for each environment can now be derived for each homogeneous group of inbred lines and the $g_{i j}^{\prime}$ values for each line regressed, independently for each group, on to these. The variation between groups can then be compared with the variation within groups for the additive genetic component and for the linear and non-linear portions of the genotype-environmental interaction component.

\section{Results}

The correlation analysis for the non-linear effects described in the last section will be illustrated by data on Nicotiana rustica taken from Experiments 1 and 2 of Perkins and Jinks (1968). In the present paper, however, both the final heights and flowering times of the lines will be considered. In order to make meaningful comparisons between seasons for flowering time, it has been scored as the number of days after sowing instead of following the usual practice of scoring from an arbitrary date (Mather and Vines, 1952). Since the final height data for Experiment 2 provides the clearest illustration of the value of the correlation analysis this will be considered first.

\section{(a) The final height for the 29 inbred varieties grown in 10 environments}

For each of the pairwise combinations of the 29 inbred varieties the correlation for the deviations from the linear regressions in each environment was computed on the KDF9 computer at Birmingham University. The correlations ranged from significantly negative values as high as -0.88 to significantly positive values as high as 0.84 . On the basis of these correlations the 29 varieties could be arranged into three groups within which all the significant correlations were positive and between which all the significant correlations were negative. The members of the three groups are listed in table 1 along with the significant pairwise correlations. The number of significant values exceeds that expected through random chance by more than a factor of two.

The two largest groups, namely 1 and 3, coincide with a well-established difference in growth habit, namely, the mop-head $(m) /$ non mop-head $(M)$ difference of Mather and Vines (1952), apart from three members of group 3 which have never been unambiguously classified for this character. The two members of group 2, like those of group 1, are non mop-head (table 1).

The between group variation is compared with the within group variation in table $2(b)$ for the additive genetic component (lines) and for the linear (heterogeneity between regressions) and non-linear (remainder) portion of the genotype-environmental interaction component. Every item in this analysis is significant (probability of $\chi^{2}<0 \cdot 1$ per cent.) when tested against the error M.S., but, what is more important, is that of the three comparisons of the between group and the corresponding within group items only that for the remainder is highly significant. This means that a marked reduction in the remainder M.S. has resulted from placing the varieties into groups on the basis of these correlations. 

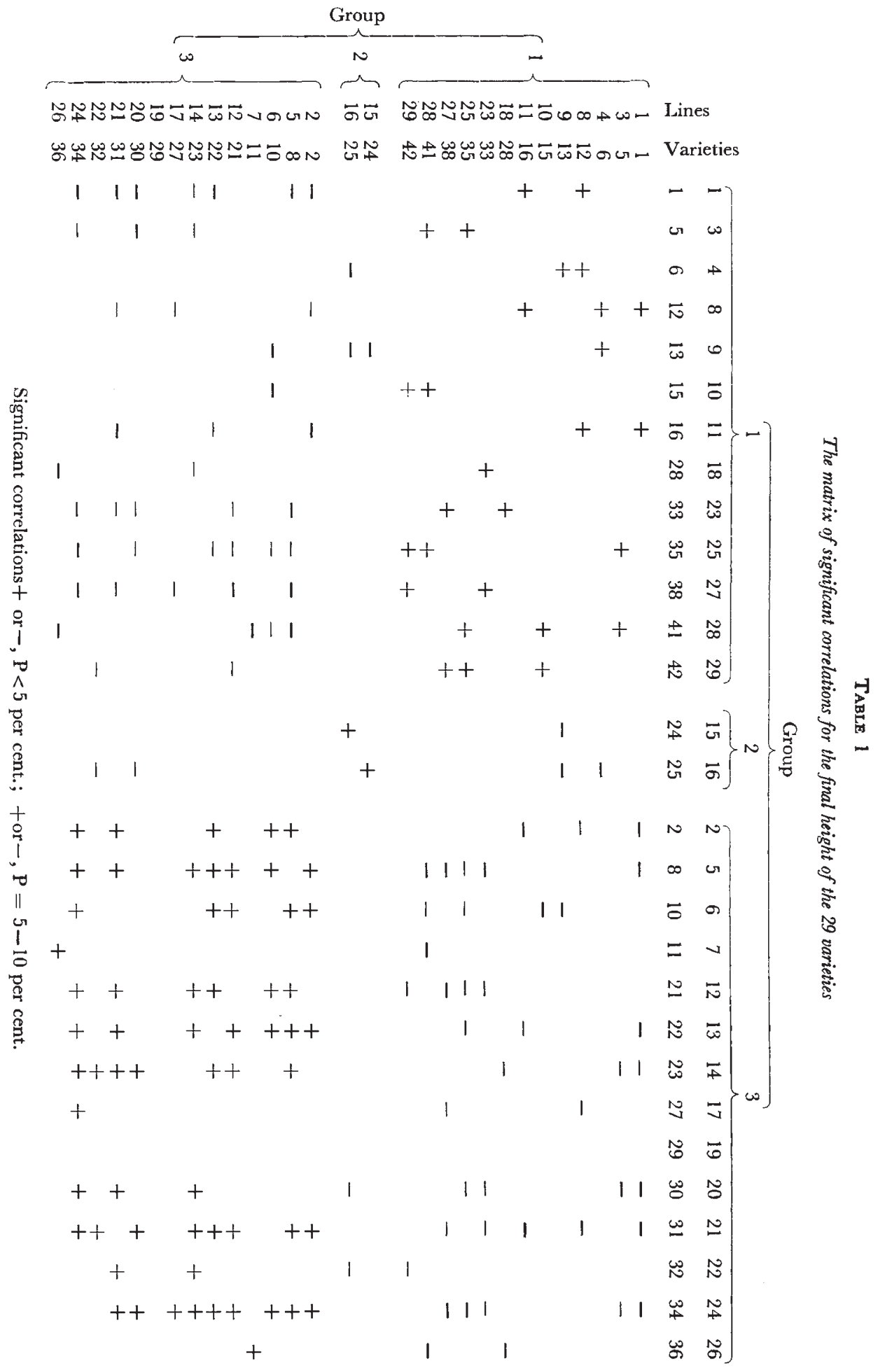

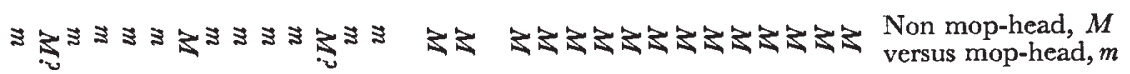


TABLE 2

Analysis of variance for the final height and flowering time of the 29 inbred varieties

(a) Joint regression analysis for flowering time

\begin{tabular}{rlrrc} 
& Item & d.f. & \multicolumn{1}{c}{ M.S. } & $\chi^{2}$ \\
& Lines & 28 & $819 \cdot 823$ & $6773 \cdot 401^{*}$ \\
G $\times \mathbf{E}$ & Environments & 9 & $1698 \cdot 465$ & $4510 \cdot 529^{*}$ \\
Int. $^{n}$ & \{et. bet. reg.'s & 28 & $28 \cdot 960$ & $239 \cdot 268^{*}$ \\
& Remainder & 224 & $24 \cdot 303$ & $1606 \cdot 348^{*}$ \\
& Error & 1599 & 3.389 & \\
& & & & $* \mathrm{P}<0 \cdot 1 \%$
\end{tabular}

Comparisons between the "between group" and " within group" items for final height and flowering time

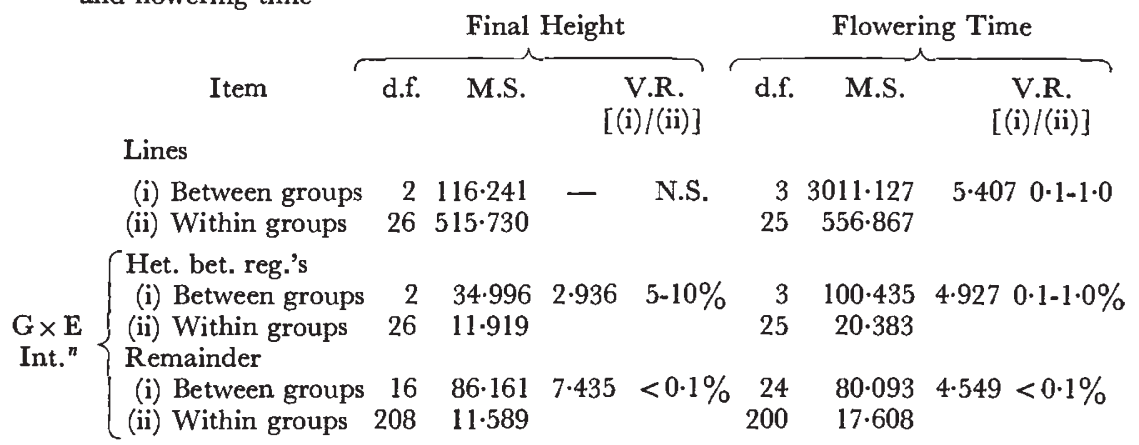

This significant reduction in the remainder M.S. is confirmed by the results of the intra-group regression analyses for the $g_{i j}^{\prime}$ values for each line against the $\epsilon_{j}^{\prime}$ values. Whereas all 29 varieties had significant remainder M.S.'s before grouping when compared against the error M.S., only 23 varieties had significant M.S.'s afterwards. Furthermore, out of the 11 lines, after grouping, which had significant linear regressions when compared against the error M.S., 6 of these accounted for a significant proportion of the variation due to genotype-environmental interaction compared with only 2 out of 15 lines before grouping (Perkins and Jinks, 1968). Nevertheless, there are still significant remainder M.S.'s which require explanation (see section 4).

Further conclusions can be drawn from the analysis of variance in table $2(b)$. The non-significance of the line item for between groups, when compared against that for within groups, means that there are no consistent differences in performance between groups. Similarly, while there is some suggestion of consistent differences in slope between groups, it is not significant ( $\mathrm{P}=5-10$ per cent.). Hence, the only recognisable difference between genotypes which coincides with the groupings is mop-head/non mop-head.

(b) Flowering time for the 29 inbred varieties grown in 10 environments

The regression analyses for flowering time, which have not been previously reported, are summarised in tables 2 and 3 . Table 3 contains the $d_{i}^{\prime}$ and $\beta_{i}^{\prime}$ values, and where the linear regression accounts for a significant portion of the genotype-environmental interaction the $\beta_{i}^{\prime}$ value is marked by an asterisk. All the items in the joint regression analysis are highly significant against the error M.S. (table $2(a)$ ). Variation among the $\beta_{i}^{\prime}$ values (hetero- 
geneity between regression) accounts for little more of the genotypeenvironmental interactions than the deviations from the linear regressions (remainder).

TABLE 3

The $d_{i}^{\prime}$ and $\beta_{i}^{\prime}$ values for the flowering time (days after sowing) of the 29 varieties in their 4 homogeneous groupings. (For the comparable values for final height see Perkins and Jinks, 1968.)

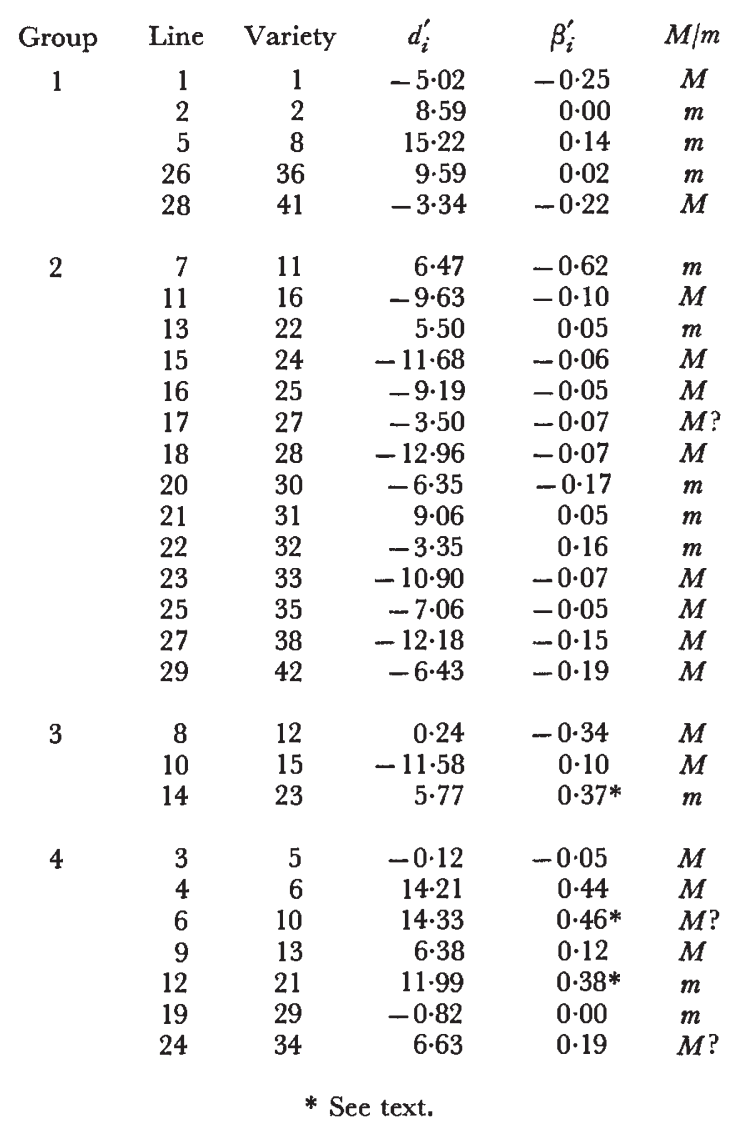

The correlation analysis was carried out, but it was found that the pattern of the significant positive and negative correlations did not allow us to make such distinct groupings as for final height. Thus, although the lines could be separated into four groups, (table 3), the smallest group (group 3), consisting of only three lines, showed one positive correlation ( $P=5-10$ per cent.) with each of two other groups (groups 2 and 4) which were themselves negatively correlated. That is, the smallest group overlapped with the two largest groups, although it could not be combined with either. Once again the number of significant correlations (64) was in excess of that expected by random chance.

None of the groups for flowering time coincides with those for final height and hence they do not correspond with the mop-head/non mop-head difference (table 3 ). The between and within group M.S.'s for lines, hetero- 
geneity between regressions and remainder are all significant when compared with the error M.S. In contrast to the corresponding analysis of final height, all the between group items for flowering time are significant against their corresponding within group M.S.'s (table 2(b)). This shows that the groups differ in their mean performances $\left(d_{i}^{\prime}\right.$ 's) and mean regression slopes $\left(\beta_{i}^{\prime \prime} \mathrm{s}\right)$ in addition to showing a significant reduction in the remainder M.S. following the separation into groups. Examination of table 3 shows that most of the lines with negative $d_{i}^{\prime}$ and $\beta_{i}^{\prime}$ values are in group 2 and most of the lines with high positive values are in group 4 . The similar pattern for $d_{i}^{\prime}$ and $\beta_{i}^{\prime}$ over groups is expected in view of the significant correlation $(r=0.49$, $\mathrm{P}=0 \cdot 1-1 \cdot 0$ per cent.) between them.

The significant reduction in the remainder M.S. (table $2(b)$ ) following the grouping of the lines, is confirmed by the individual intra-group regression analyses. Thus while 28 lines showed significant remainder M.S.'s before grouping, only 22 lines did so after grouping. Nevertheless there are still significant remainder M.S.'s that require explanation (section 4). For the significant linear regressions, 4 out of 15 accounted for all or most of the genotype-environmental interaction before grouping and 5 out of 11 did so afterwards. Thus for flowering time there is no marked improvement in the number of lines having linear regressions which account for a significant proportion of the variation due to genotype-environmental interaction, even though there is a general reduction in the remainder M.S. values after grouping. This can be attributed to a decreased heterogeneity between regressions within groups owing to the similarity of the $\beta_{i}^{\prime}$ values within them (tables $2(b)$ and 3$)$.

\section{(c) The final height for the 20 inbred varieties grown in 9 environments}

The twenty lines can be divided into four groups on the basis of the significant correlations between them, although one member of each of two of the groups are significantly correlated. The analysis of variance for between and within group comparisons is given in table $4(b)$. All the items in this analysis are highly significant against the error M.S. except for the heterogeneity of regression between groups $(P=20-30$ per cent.). Apart from the latter, the picture is identical with that for final height in the previous experiment in that the only significant between group item when tested against the corresponding within group item is that for the remainder M.S. Hence, separating the lines into groups, which are internally homogeneous, has only achieved a reduction in the size of the regression remainder M.S. There are no consistent differences between the groups either for mean performance or for regression slope. Furthermore, the groups do not correspond with the only major gene difference (anthocyanin, A/anthocyaninless, a) which distinguish the inbred lines. Examination of the intra-group regression analyses for the individual lines shows that after grouping there is one additional significant regression, making a total of 5, that accounts for all or most of the genotype-environmental interaction.

\section{(d) Flowering time for 20 inbred lines grown in 9 environments}

The regression analyses for flowering time, which have not been previously reported, are summarised in tables $4(a)$ and 5 . Table 5 contains the $d_{i}$ and $\beta_{i}$ values and where the latter accounts for a significant portion of the genotype-environmental interaction the $\beta_{i}$ value is marked by an 
asterisk. All the items in the joint regression analysis are highly significant against the error M.S. (table $4(a)$ ). Variation among the $\beta_{i}$ values (heterogeneity between regression) accounts for most of the genotype-environmental interactions and is significant against the remainder M.S.

TABLE 4

Analyses of variance for the final height and flowering time of the 20 inbred lines

(a) Joint regression analysis for flowering time.

\begin{tabular}{|c|c|c|c|c|c|}
\hline & Item & d.f. & M.S. & $\begin{array}{c}\chi^{2} \\
\text { (against 4) }\end{array}$ & $\begin{array}{c}\chi^{2} \\
(2 / 3)\end{array}$ \\
\hline & Lines & 19 & $232 \cdot 962$ & $1951 \cdot 625^{*}$ & \\
\hline & 1. Environments & 8 & $1336 \cdot 547$ & $4714 \cdot 451^{*}$ & \\
\hline $\mathrm{G} \times \mathrm{E}$ & 厅 2. Het. bet. reg.'s & 19 & $24 \cdot 784$ & $207 \cdot 628^{*}$ & $63 \cdot 447 *$ \\
\hline \multirow[t]{2}{*}{ Int. ${ }^{n}$} & 3. Remainder & 133 & $7 \cdot 422$ & $435 \cdot 215^{*}$ & \\
\hline & 4. Error & 957 & $2 \cdot 268$ & & \\
\hline
\end{tabular}

(b) Comparisons between the " between group" and " within group" items for final height and flowering time.

\begin{tabular}{|c|c|c|c|c|c|}
\hline & Fin & ight & & Flow & time \\
\hline tem & d.f. M.S. & $\begin{array}{l}\text { V.R. } \\
{[(\mathbf{i}) /(\text { ii) }]}\end{array}$ & d.f. & M.S. & \\
\hline
\end{tabular}

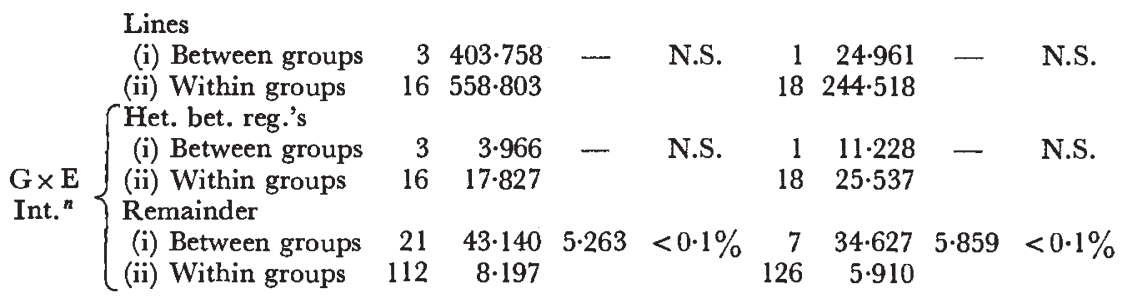

The twenty lines can be divided into two clear-cut groups on the basis of the significant correlations between them. These groups do not coincide with the only known major gene difference which distinguish them $(A / a)$. The analysis of variance for the within and between group comparisons is given in table $4(b)$. All the items in this analysis are significant against the error M.S., but like final height the only significant between group item when tested against the corresponding within group item is the remainder M.S. Hence once again the groups do not differ in their mean performance or mean regression slope and the only effect of the grouping is a reduction in the regression remainder M.S.

Examination of the intra-group regression analyses for individual lines confirms that there is a reduction in the remainder M.S. in that before grouping there are 11 lines with significant remainder M.S.'s whereas there are only 7 after grouping. Before grouping there was a very high proportion of lines, namely one-half, which had significant regressions that accounted for all or most of the genotype-environmental interaction. It is not surprising therefore, that grouping produced no increase in this proportion. 
TABLE 5

The $d_{i}$ and $\beta_{i}$ values for the flowering time of the 20 inbred lines in their 2 homogeneous groupings. (For the comparable values for final height see Perkins and Finks, 1968.)

\begin{tabular}{cccc} 
Group & Line & \multicolumn{1}{c}{$d_{i}$} & $\beta_{\boldsymbol{i}}$ \\
1 & 1 & $-2 \cdot 50$ & $-0 \cdot 22^{*}$ \\
& 2 & $1 \cdot 85$ & $-0 \cdot 16$ \\
& 4 & $2 \cdot 96$ & $0 \cdot 11$ \\
& 11 & $-6 \cdot 22$ & $0 \cdot 00$ \\
& 12 & $-2 \cdot 21$ & $-0 \cdot 15^{*}$ \\
& 13 & $-1 \cdot 30$ & $0 \cdot 00$ \\
& 15 & $3 \cdot 63$ & $0 \cdot 07$ \\
& 16 & $2 \cdot 35$ & $-0 \cdot 04$ \\
2 & 20 & $-2 \cdot 25$ & $-0 \cdot 30^{*}$ \\
& 3 & $-1 \cdot 30$ & $-0 \cdot 19 *$ \\
& 5 & $-6 \cdot 25$ & $0 \cdot 06$ \\
& 6 & $-5 \cdot 43$ & $0 \cdot 38^{*}$ \\
& 7 & $1 \cdot 01$ & $-0 \cdot 20$ \\
& 8 & $-1 \cdot 89$ & $0 \cdot 09$ \\
& 9 & $2 \cdot 71$ & $-0 \cdot 17 *$ \\
& 10 & $-3 \cdot 44$ & $0 \cdot 14^{*}$ \\
14 & $5 \cdot 64$ & $0 \cdot 11$ \\
17 & 16.43 & $0 \cdot 61^{*}$ \\
18 & -2.74 & $-0 \cdot 03$ \\
& 19 & $-1 \cdot 04$ & $-0 \cdot 11^{*}$ \\
& & &
\end{tabular}

\section{THE NON-LINEAR GOMPONENT OF} GENOTYPE-ENVIRONMENT INTERACTION

In every set of data examined an overall reduction in the non-linear portion of the variation due to genotype-environmental interaction (regression remainder) has resulted from placing the lines into groups such that the members of the same group are positively correlated for their deviations $\left(\delta_{i j}\right)$ from their linear regressions. Even after grouping, however, a significant non-linear portion of the variation due to genotype-environmental interaction remains. Furthermore, the magnitude of the remainder M.S. appears to be a characteristic of the genotype since in general there is a significant positive correlation in their values before and after grouping. That is, grouping of the lines leads to an overall reduction in the remainder M.S.'s but no change in their relative magnitudes among the genotypes. Thus some lines have characteristically large deviations from their regression, both before and after grouping, while others have consistently small deviations.

The differences in the magnitudes of the remainder M.S.'s are in no way related to the relative mean performance $\left(d_{i}^{\prime}\right)$, to the sensitivity of the genotypes to environmental differences as measured by the linear regression slope $\left(\beta_{i}^{\prime}\right)$, or to the magnitudes of the variation within environments except for flowering time among the 20 inbred lines of experiment 1 . Even in the latter case the correlation was due entirely to one line, number 17 , which had the highest values for $d_{i}, \beta_{i}$, the remainder M.S. and the within environmental variation. In general, therefore, the linear and non-linear component of the genotype-environmental interaction and the within environ- 
mental variation not only differ among genotypes, but they do so independently. Hence, they are presumably under the control of different genetic systems.

\section{Discussion}

In the present and previous papers in this series three characteristics of the phenotype have been considered. These are the additive genetic component, $d_{i}^{\prime}$, and the linear, $\beta_{i}^{\prime}$, and non-linear, $\delta_{i j}$, portions of the interaction between the genotype and the environment. For some combinations of phenotypes and environments the linear portion accounts for all or most of the interaction between them. However, in other cases most of the interaction is accounted for by the non-linear component. It is quite clear from the results of our re-analysis after grouping that part, but not all, of this nonlinear component can be attributed to the extreme diversity of the genotypes. The relative magnitudes of the non-linear component for the different genotypes is however unchanged by grouping, which implies that this component is as much a characteristic of the genotype as the additive genetic and linear components. Except for the character final height in experiment 2 the within environmental component of variation also differs significantly among genotypes.

All of these genotypic attributes are with two exceptions independent. The first exception is the positive correlation between $d_{i}^{\prime}$ and $\beta_{i}^{\prime}$ for final height in experiment 2 (Perkins and Jinks, 1968) and for flowering time in both experiments, being significant at the 5 per cent. level in experiment 1 and at the $0 \cdot 1-1.0$ per cent. level in experiment 2 . The second exception is the correlation between the non-linear component and the within environmental variation for flowering time in experiment 1 which can be traced to one extreme line. For the genotypes of experiment 1, which were derived by inbreeding without selection from an initial cross between two inbred lines, we can estimate the number of genes (effective factors) controlling the variation in $d_{i}$ and $\beta_{i}$. Thus, within the usual assumptions (Mather, 1949), half the difference between the two extreme inbred lines for any character equals $k d$ and the genetic variance of the inbreds equals $k d^{2}$, where $k$ is the number of genes. Hence we can derive $k$ as $\frac{(k d)^{2}}{k d^{2}}$. For final height and flowering time the estimates of $k$, which are of course minimal, are 4 and 5 for $d_{i}$ and 5 and 5 for $\beta_{i}$ respectively. The correlations between $d_{i}$ and $\beta_{i}$ are approximately 0.4 for both characters. Hence, if pleiotropy is responsible for these correlations the two aspects of the genotype, $d_{i}$ and $\beta_{i}$, must have less than one gene in common for both final height and flowering time.

We can further consider the relationships between the corresponding $d_{i}$ and $\beta_{i}$ values of the two characters. These are significant only for $d_{i}$ in both experiments. For experiment 1 the correlation is $0 \cdot 84$, which means that if pleiotropy is responsible there are three genes in common controlling the $d_{i}$ values for final height and flowering time. For experiment 2 the correlation is 0.51 which means that 25 per cent. of the genes could be in common. Only the analysis of segregating generations could confirm whether or not pleiotropy rather than linkage or chance association is responsible for the significant correlations, but irrespective of the outcome of such an analysis there is sufficient independent variation between the different levels of the 
interaction between genotype and environment to allow considerable reassortment by selective breeding.

\section{Summary}

1. While a significant proportion of the genotype-environmental component of variation is a linear function of the additive environmental component, there is frequently a significant remainder that is not.

2. On the basis of the significant positive and negative correlations for the deviations from the linear regressions, the lines can be separated into groups such that all the positive correlations occur between members of the same group and the negative correlations between members of different groups.

3. In every set of data examined an overall reduction in the non-linear portion of the variation due to genotype-environmental interaction resulted from separating the lines into groups.

4. After grouping, however, a significant non-linear portion of the interaction remained. Furthermore, positive correlations were found between the regression remainder M.S. values of the lines before and after grouping. Hence their relative magnitudes are presumably determined by the genotype of the lines.

5. In general the groups do not coincide with the measurable differences among the lines. For final height in experiment 2, however, the two larger groups correspond with a difference in growth habit controlled by a single gene $(M: m)$, and in the same experiment there is a correlation between the groups and their mean performances and mean linear regression slopes for flowering time.

6. Of the four components of the phenotype, namely, mean performance, linear and non-linear components of the genotype-environmental interaction, and the within environment component, only the first two show significant, though small, correlations for both characters in both experiments. In general, therefore, the various components are independent and presumably subject to the control of different genetic systems.

Acknowledgments. - This work was supported by a Grant from the Agricultural Research Council.

\section{REFERENCES}

MATHER, K. 1949. Biometrical Genetics. The study of Continuous variation. Dover Publications Inc.

MATHER, K., AND VINEs, A. 1952. The inheritance of height and flowering time in a cross of Nicotiana rustica. from " Quantitative Inheritance", ed. E. C. Reeve and C. H. Waddington. H.M.S.O., London, pp. 49-80.

PERKINS, JEAN M., AND JINKS, J. L. 1968. Environmental and genotype-environmental components of variability. III. Multiple lines and crosses. Heredity, 23, 339-356. 